\title{
Detection of Mixed OAM States via Vortex Breakup
}

\author{
Mariia Shutova*, Alexandra A. Zhdanova, Alexei V. Sokolov* \\ The Institute for Quantum Science and Engineering, Physics and Astronomy Department, \\ Texas A \& $M$ University, 4242 TAMU, College Station, TX 77843, USA
}

\begin{abstract}
We study the tilted lens technique for measuring the topological charge (TC) of an optical vortex and investigate how this technique works for optical vortices in mixed orbital angular momentum states (i.e. when one beam contains several components with different values of TC). We present experimental results and theoretical simulations for the measurement of the TC of mixed states. We investigate two different cases: when coherent interference (or addition) between components is present and when it is absent (incoherent addition). We discover that this technique is suitable for measuring the TC of the dominant component of a mixed state.
\end{abstract}

Keywords: optical vortices, topological charge, mixed OAM states

\section{Introduction}

The optical vortex $(\mathrm{OV})$ is a light beam with a donut-shaped transverse profile and a spiral wavefront. Its phase exhibits a singularity in the center (where the intensity is zero) and carries an amount of orbital angular momentum ${ }_{5}(\mathrm{OAM})$ per photon equal to $\hbar l$, where $l$ is the topological charge (TC) of the OV 1]. The OAM properties of OVs are actively being studied because of their many applications, such as free space telecommunications [2, mode division multiplexing in a fiber [3, 4] optical micro- and nanomanipulation [5, 6, 7], astronomy [8] and many others. Since measurement of the TC is the common

\footnotetext{
* Corresponding author

Email address: mariia.shutova@physics.tamu.edu (Mariia Shutova)
}

Preprint submitted to Physics Letters A

September 6, 2016

(C) 2016. This manuscript version is made available under the Elsevier user license http://www.elsevier.com/open-access/userlicense/1.0/ 
inevitable link in studying these processes, it is very important to characterize the standard measurement techniques thoroughly.

There are a variety of methods for measuring the TC of OVs; some are based on interferometry [9, 10, 11, others use an annular [12] or triangular aperture [13. Methods based on the property of multi-singular optical vortices to deform 15 in the presence of some astigmatic media are particularly relevant to experimental use because of their extreme simplicity. Astigmatic laser beam behavior, propagation, and transformation were studied theoretically and experimentally in some detail for both Bessel modes of light [14], and Laguerre-Gaussian [15, 16] ones. The invention of a rather simple Hermite to Gaussian (and vice versa) mode converter exploiting a cylindrical lens to add astigmatism into the beam [17, 18] evolved into several techniques which allow experimentalists to measure the topological charge of OVs very easily [19, 20]. In this work, we study this last category of techniques, where we add astigmatism into the beam by inserting a tilted plano-convex lens; this technique is referred to throughout the manuscript as the "titled lens technique". This method has successfully been shown to measure the magnitude and sign of an OV with TC up to $|l|=14[20$.

In the current work, we investigate the possibility of using a tilted convex lens to detect the mixed OAM state 21] and measure its TC. This means that the OAM state of the OV may not necessarily be a pure state with a single TC 30 value across the whole beam, but may contain several components with different OAM states and corresponding values of TC. These states arise as a result of flaws in the optical devices, such as spatial light modulators (SLM) or computergenerated holograms (CGH), widely used for generating OVs. In practice, these devices create wavefronts which differ from the ideal and may have distorted phase and amplitude [22, 23]. Because SLMs and CGHs are phase-only modulators, these small distortions can be interpreted as separate components of the beam with different TCs (since TC is the only parameter which characterizes phase). Because nonlinear process amplify small perturbations (noise) [24, 25, these extra components play a large role for OVs in different nonlinear processes 40 such as second harmonic generation [26], Raman generation with femtosecond 

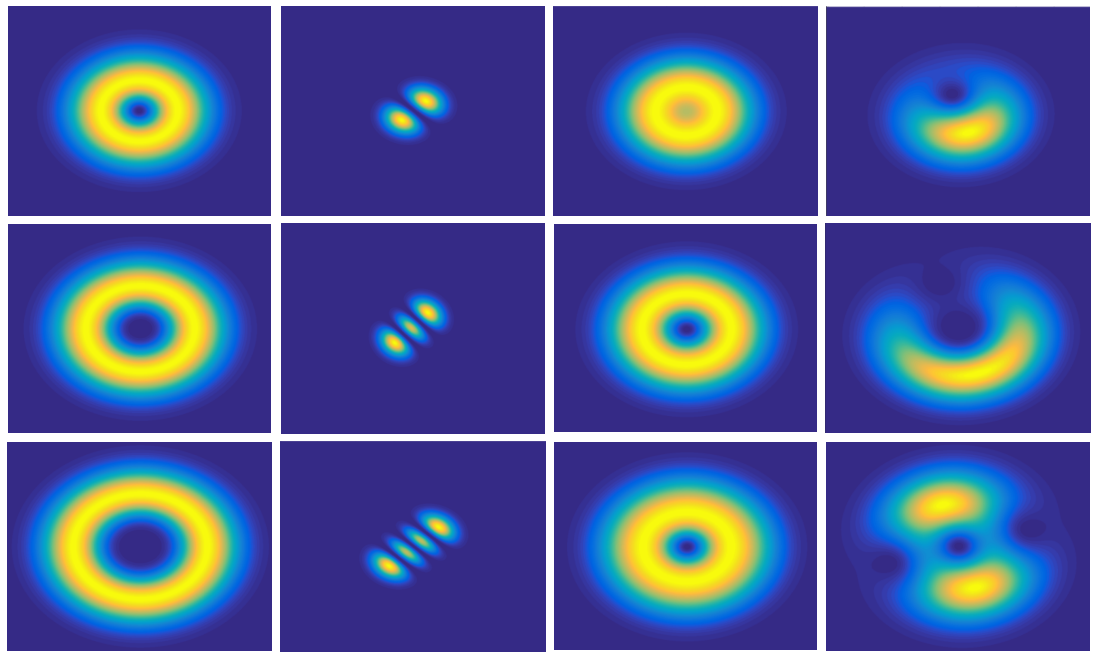

Figure 1: Transverse intensity distribution of OVs (color online). First column - pure OVs with $l=1$ (top); $l=2$ (middle); $l=3$ (bottom). Second column - pure OVs at the focal plane of tilted lens with $l=1$ (top); $l=2$ (middle); $l=3$ (bottom). Third and fourth columns - incoherent and coherent mixed states with $50 \%$ of each component in every OV, correspondingly, $\mathrm{LG}_{00}+\mathrm{LG}_{01}$ (top); $\mathrm{LG}_{01}+\mathrm{LG}_{02}$ (middle); $\mathrm{LG}_{01}+\mathrm{LG}_{03}$ (bottom).

OVs [27, 28, 29], or white light generation [30]. As a result, the nonlinearly generated beams may become so distorted that they represent a mixed state 31] containing comparable amounts of different OAM values.

This mixed state may be coherent - when components do interfere with 45 each other [32] or incoherent - when components do not interact with each other. When the mixed state is coherent, it is possible to detect it by observing the OV's transverse profile, the so called off-axial vortex [33]. However, the detection of incoherent mixtures by observing the transverse profile is less obvious. Examples of coherent and incoherent mixtures $\mathrm{LG}_{00}+\mathrm{LG}_{01}, \mathrm{LG}_{01}+\mathrm{LG}_{02}$, $\mathrm{LG}_{01}+\mathrm{LG}_{03}$ are illustrated in Fig. 1. As has been shown by Khonina et al. in 34], coherent superpositions have characteristic "chamomile-shaped" transverse intensity distribution profiles. Several algorithms are known to analyze the composition of mixed OAM states. Some techniques based on exploiting diffractive optical elements (DOE) have also been developed to separate OVs 
with different TCs in free-space. These techniques utilize the property of beams composed of components with different OAMs to "drift" from the optical axis at different distances [35, 34, 36, 37]. The use of a multistage method to determine the TC of a single photon is also possible; this method is based on using rotated dove prisms and a Mach-Zehnder interferometer in each stage [38]. In

60 this letter, our group aims to study the applicability of the tilted lens technique, which is fairly simple in use and does not demand the separation of different components in space, to detect a mixed OAM state. The possibility of analyzing the composition of this state and distinguishing between coherent and incoherent mixed states is also investigated and discussed.

\section{Theory}

Mathematically, an optical vortex beam propagating along the $z$ axis in cylindrical coordinates (also known as a Laguerre-Gaussian or LG beam) can be represented by a complex field:

$$
\begin{aligned}
E(\rho, \phi, z, t)= & E_{0} \frac{w_{0} \rho^{|l|}}{w^{|l|+1}} \exp \left[\frac{-\rho^{2}}{w^{2}}\right] \\
& \times \exp \left[-i(|l|+1) \arctan \frac{z}{z_{R}}+\frac{i k \rho^{2}}{2 R(z)}+i(l \phi+k z-w t)\right]
\end{aligned}
$$

where $\rho, \phi$ are polar coordinates in the transverse plane; $w_{0}$ is the radius 70 of the beam at the beam waist (at $z=0$ ); $E_{0}$ is amplitude parameter; $w=$ $w_{0}\left(1+z^{2} / z_{R}^{2}\right)^{1 / 2}$ is the transversal beam radius; $z_{R}=k w_{0}^{2} / 2$ is the Rayleigh range; $R(z)=z\left(1+z_{R}^{2} / z^{2}\right)$ is the radius of the wavefront curvature; $l$ is the value of the TC; $k$ is the wavenumber [25]. The complex amplitude of an OV at the beam waist in Cartesian coordinates can be written as follows:

$$
E(x, y)=(x \pm i y)^{l} \exp \left[-\frac{x^{2}+y^{2}}{w_{0}^{2}}\right]
$$

At a certain distance from the beam waist in the presence of an astigmatic lens, a higher order OV breaks into $|l|$ secondary first-order vortices [39, 32. In 
the transverse plane at this distance there will be $|l|+1$ intensity peaks, oriented at a $\pm 45^{\circ}$ angle relative to the tilt axis [15. The propagation dynamics of an $\mathrm{OV}$ in the presence of a tilted spherical lens can be simulated using a ray transfer matrix analysis and generalized Huygens-Fresnel integral, as related in detail in [20]. In the end, the complex amplitude may be described as:

$$
E=f_{1} \exp \left[-\left(f_{2} x^{2}+f_{3} y^{2}\right)\right] H_{l}\left(f_{4} x^{2}+f_{5} y^{2}\right)
$$

where $x, y$ are transverse Cartesian coordinates. $f_{1}, \ldots f_{5}$ are the complex functions of $\theta$ (tilt of the lens), $z, z_{0}$ (distance from the waist plane to the tilted lens), $f$ (focal distance of the tilted lens) and $w_{0}$, described as follows:

$$
\begin{gathered}
f_{1}=\frac{i^{m+1}}{2} \quad \frac{k w_{1} w_{2}\left(w_{1}^{2}-w_{2}^{2}\right)^{m / 2}}{\left(b_{1} b_{2}\right)^{1 / 2}} ; \quad f_{2}=\left(\frac{k w_{1}}{2 b_{1}}\right)^{2}+i \frac{k d_{1}}{2 b_{1}} \\
f_{3}=\left(\frac{k w_{2}}{2 b_{2}}\right)^{2}+i \frac{k d_{2}}{2 b_{2}} ; \quad f_{4}=\frac{k w_{1}^{2}}{2 b_{1}\left(w_{1}^{2}-w_{2}^{2}\right)^{1 / 2}} ; \quad f_{5}=\frac{i \operatorname{sign}(l) k w_{2}^{2}}{2 b_{2}\left(w_{1}^{2}-w_{2}^{2}\right)^{1 / 2}} .
\end{gathered}
$$

Where:

$$
\begin{aligned}
b_{1} & =z_{0}+z-\frac{z z_{0} \sec \theta}{f} & b_{2} & =z_{0}+z-\frac{z z_{0} \cos \theta}{f} \\
d_{1} & =1-\frac{z_{0} \sec \theta}{f} & d_{2} & =1-\frac{z_{0} \cos \theta}{f} \\
w_{1}^{2} & =\frac{2 b_{1} w_{0}^{2}}{2 b_{1}+i k\left(1-\frac{z}{f} \sec \theta\right) w_{0}^{2}} & w_{2}^{2} & =\frac{2 b_{2} w_{0}^{2}}{2 b_{2}+i k\left(1-\frac{z}{f} \cos \theta\right) w_{0}^{2}}
\end{aligned}
$$

At this point, the complex field is simply an elliptic Gaussian field modulated by a Hermite polynomial [20]. For a mixed OAM state, the complex field is a superposition of $n$ fields:

$$
E=A E_{l_{1}}+B E_{l_{2}}+C E_{l_{3}}+\ldots+D E_{l_{n}}
$$

where $l_{1}, l_{2}, l_{3}, \ldots l_{n}$ are topological charges corresponding to each field, and $A, B, C, \ldots D$ are the weight coefficients for each field. In this letter, we 90 consider the superposition of two fields, $E=A E_{l_{1}}+B E_{l_{2}}$. However, it is easy 
to extend this theory to the superposition of three or more fields. The intensity of the resultant field superposition can be found as follows:

$$
I=|E|^{2}=A^{2}\left|E_{1}\right|^{2}+B^{2}\left|E_{2}\right|^{2}+\alpha\left(A B E_{1} E_{2}^{*}+A B E_{2} E_{1}^{*}\right)
$$

The third term represents the coherent contribution to the intensity distribution, where $\alpha$ is a positive numerical coefficient signifying a degree of coherence

95 incoherent ( $\alpha=0$, keeping only 1st and 2nd terms);2) completely coherent ( $\alpha=1$, keeping all terms); 3) partially coherent $(0<\alpha<1)$. This work focuses on the first and second cases because one can imagine the third case as a linear combination of the first two cases. We calculate the intensity distribution near for all the states that we included in the Supplementary Materials, it is not the $\mathrm{TC}$ of each component which plays a significant role in this evolution, but the difference between the TCs of each component $(\Delta l)$ which does. In other words, let us take any mixed OAM state with two components such that the difference split into two in the coherent case and an additional peak will emerge from the center in the incoherent case (see Fig. 2, (a) ). Meanwhile, for any combination with $\Delta l=2$, we will get no difference between the coherent and incoherent cases 
- each of the two outside peaks will split into two (Fig. 2, (b) ).

Hence, we note that different evolutionary mechanics for coherent/incoherent addition of states are at play for TC differences equal to 1 and those equal to 2. This difference extends to other cases of $\Delta l$ as well $(\Delta l=3,4, \ldots$ each have their own evolution). Further examples to support our assertion are available in the Supplementary Materials. We also can conclude that by observing the transverse intensity distribution, we can detect the mixed OAM state and distinguish between coherent and incoherent cases. Moreover, it can be seen from Fig. 2 that the tilted lens technique is insensitive to the perturbations (up to $\sim 30 \%$ ) around a pure state and indicates only the dominant component of a mixed state.

Finally, we find that the tilted lens technique can distinguish between different ratios of "mixedness" in the mixed states, but only by measuring the relative intensities of each peak in the transverse intensity distribution. Particularly in the cases when an equal amount of each TC component is present, the intensity distribution largely deviates from the ideal case - the peaks can no longer be clearly distinguished in some cases, while in others they are so small as to be barely visible.

\section{Experiment}

A schematic of the experimental setup is shown in Fig. 3. The beam from a CW He-Ne laser $(\sim 0.7 \mathrm{~mW}, 632 \mathrm{~nm})$ was split by a $50 \mathrm{x} 50$ beam splitter into two equal components. After splitting, each component was shaped into a pure OAM state by an SLM (Hamamatsu x10468-02). After traveling $z_{0}=2 \mathrm{~m}$ from the laser output plane, which we considered to be the beam waist plane [40], the beams were recombined coherently or incoherently to create a mixed OAM state. In the "incoherent" mixing case, there were several measures taken to minimize coherent interference. For example, one beam was delayed by longer than the coherence length with respect to the other (but care was taken to keep the diameter of both vortices nearly equal) and a polarizer was used to 


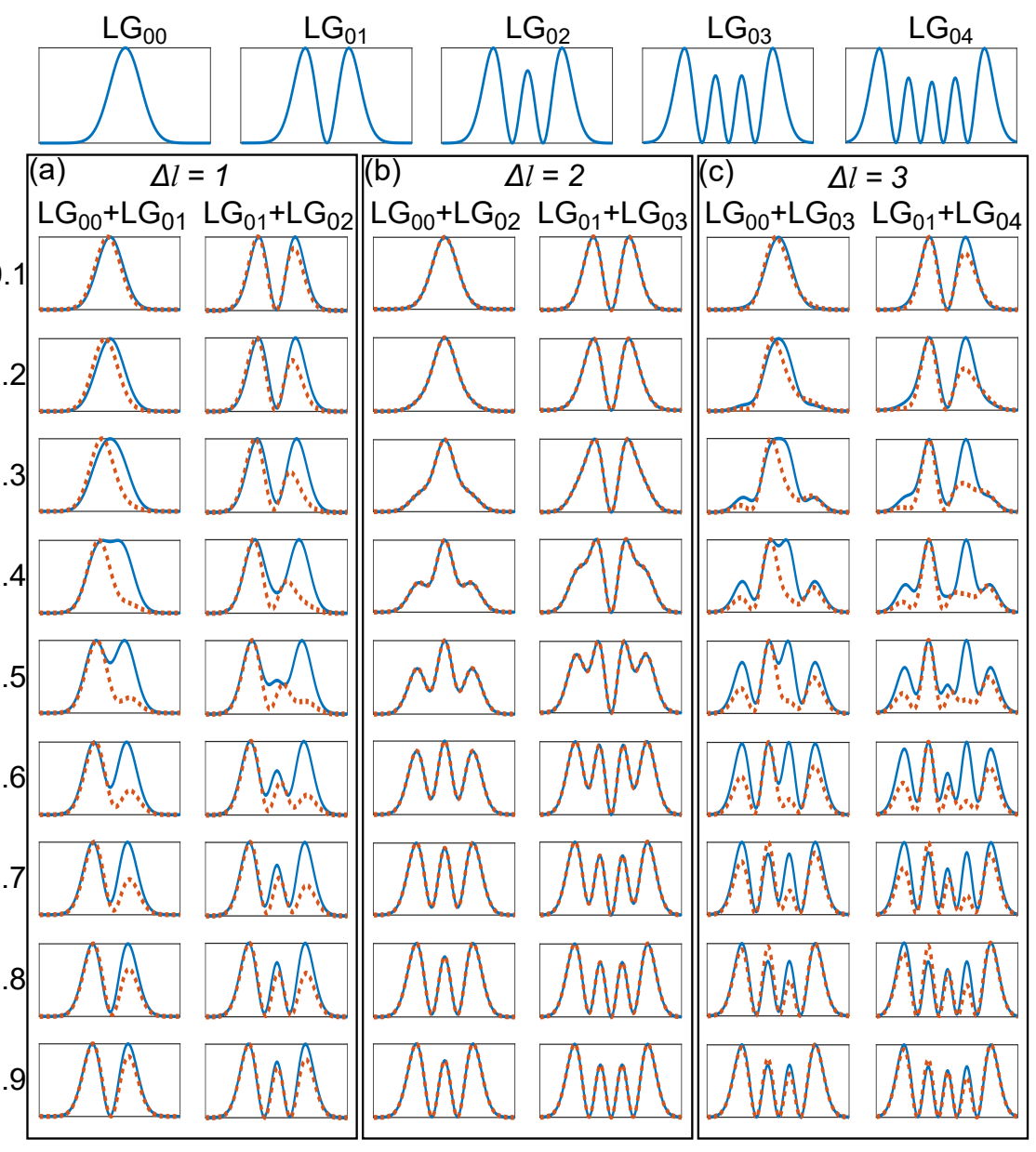

Figure 2: Theoretical intensity distribution near the focal point of a tilted lens along the $+45^{\circ}$ direction in the transverse plane of the optical vortex for mixed OAM states. The numbers on the left signify the weight of the component with the highest topological charge. Top-most graphs indicate pure OAM states for $\mathrm{LG}_{00}$ (Gaussian beam), $\mathrm{LG}_{01}, \mathrm{LG}_{02}, \mathrm{LG}_{03}$, and $\mathrm{LG}_{04}$. The three boxes - (a), (b), (c) - show the cross section of the intensity evolution of the mixed OAM states with different values of $\Delta l$, varying the weight coefficients of each OV in the mixed state. The blue solid line indicates the incoherent addition of two components of the state, the orange dotted line indicates coherent addition (color online). (a): in the coherent case, as the amount of $\mathrm{LG}_{01}\left(\mathrm{LG}_{02}\right)$ increases, the right peak starts to decrease, moves left and splits into two at the point of equality of each component so that in the end (a nearly pure state) the intensity distribution is indistinguishable from that of $L_{01}\left(L_{02}\right)$; for the incoherent addition the intensity evolution is symmetric and the middle peak appears in-between the two initial ones. (b): coherent and incoherent addition are indistinguishable. (c): additional peaks emerge from the left and right from the center peak, which remains almost unchangeable. 


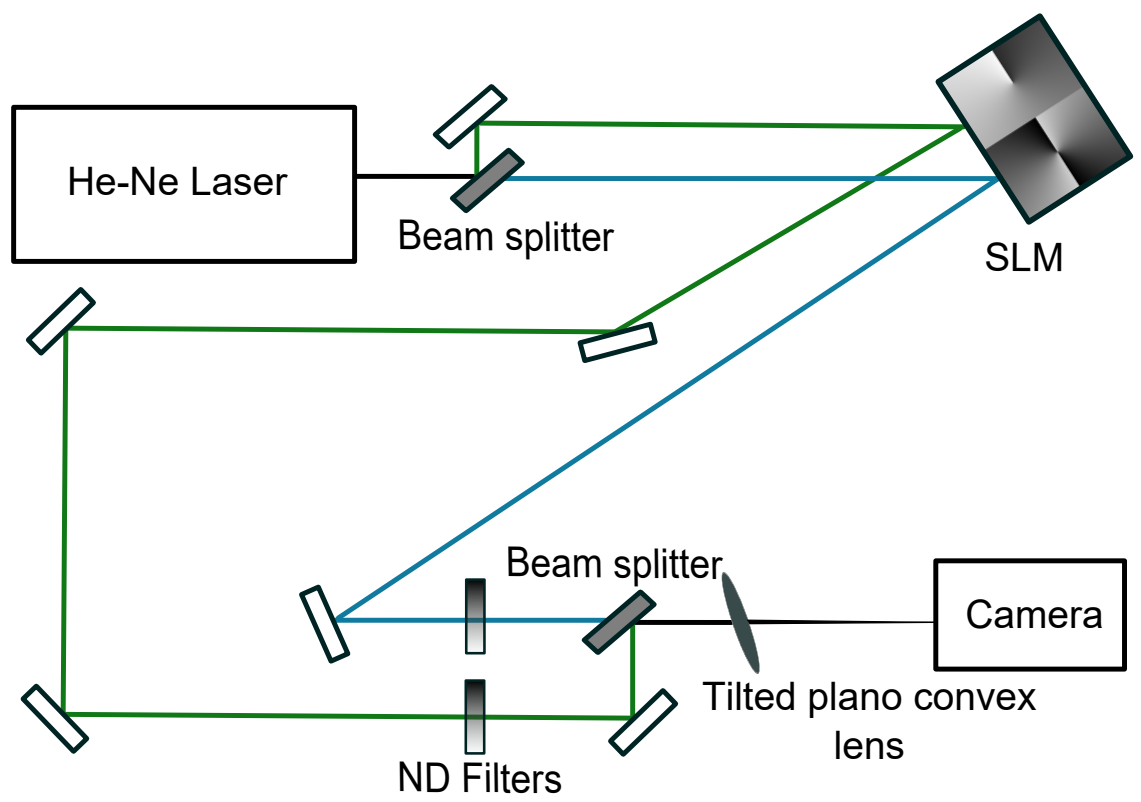

Figure 3: Experimental setup for measuring the topological charge of mixed OAM states. The angle of the SLM is greatly exaggerated (color online).

ensure that the polarizations of the two beams were crossed, further preventing interference. In the coherent case, the same experiment was carried out without delaying the second component or using a polarizer.

In all cases, the recombined beam was then focused by a tilted (at an angle $\theta \approx 11^{\circ}$ ) convex lens of focal length $f=20 \mathrm{~cm}$ in the manner described in the introduction. The results were then collected by a beam profiler (Spricon SP-620 U) near the focal point of the lens $(z \approx f \approx 20 \mathrm{~cm})$. Variable neutral density (ND) filters (Thorlabs NDC-50C-4M) were used to change the relative intensities of each component. These results are summarized in Fig. 4. For clarity, experimental results are depicted along with the theoretical two-dimensional intensity distributions; good agreement between simulation and experiment can be seen from these results. 

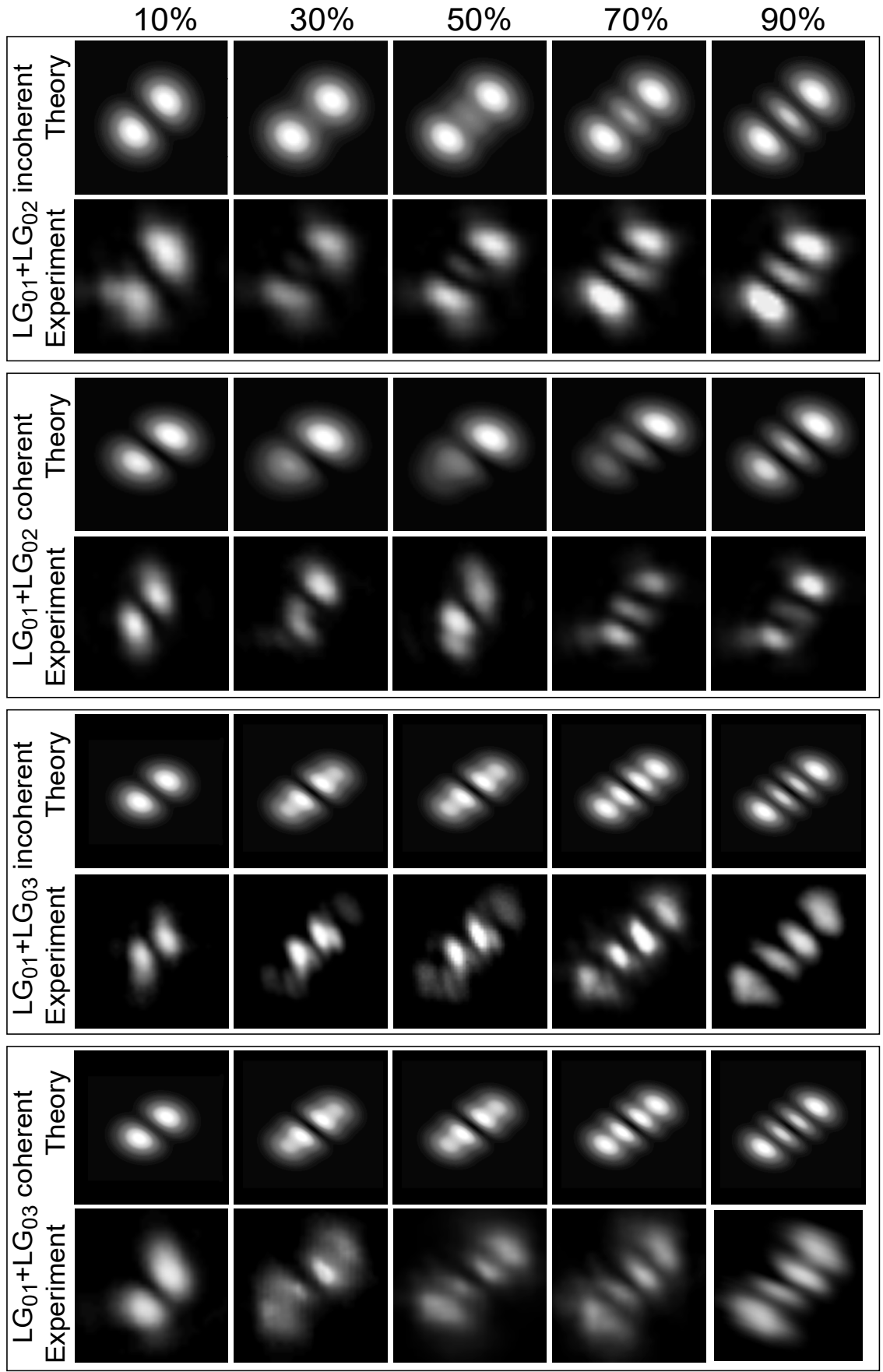

Figure 4: Intensity distribution near the focal point of the tilted lens for mixed OAM states; the relative intensity of the optical vortex with the highest topological charge is indicated. 


\section{Discussion}

The results of our theoretical and experimental work show that the tilted lens technique can be used to detect a mixed OAM state (superposition of two components). As shown in Fig. 4 for $\mathrm{LG}_{01}+\mathrm{LG}_{02}$, both in the coherent and incoherent cases the experimental implementation of the tilted lens technique showed all of the theoretically predicted principal differences between coherent and incoherent addition of components (the splitting of one spot for coherent addition and the emergence of a third spot in between the central two for incoherent addition). Moreover, this technique may be used to analyze the composition of the beam by observing the evolution of the transverse intensity distribution without the separation of different components in space when both components have relatively equal weight coefficients. We have shown that the experimental intensity distribution is consistent with that calculated using Eqn. 5. For relatively pure OAM states (dominant component is $70 \%$ or more) the technique yields the $\mathrm{TC}$ of the dominant component of the beam. When the beam contains several (two or more) components with equal intensity, the tilted lens technique detects a mixed OAM state and shows whether coherent or incoherent addition of the components is present. In this case, a deeper analysis or an alternate technique discussed in the Introduction to this letter is needed to obtain the TC for such a mixed OAM state.

\section{Conclusions}

To summarize, the tilted lens technique is easy technique which can be practically used for detecting mixed OAM state, although, it cannot be used to determine the TC and weight coefficient of every component of this mixed state, but it can show that a certain OAM state is not pure and may contain several equal components with different topological charge. This fact can be used as a starting point for applying other, less simple algorithms mentioned above to analyze the composition of the beam and to determine the OAM of the whole beam. Moreover, we conclude that the resultant TC determined with 
this method is rather insensitive to the interference between components and

gives the same number of spots (intensity peaks) in the cases of interfering and non-interfering components. However, the mechanism for the evolution of these peaks and their respective intensities is very different in the cases of coherent and incoherent addition. Hence, a separate treatment is needed for each case.

\section{Acknowledgments}

This work was supported by the National Science Foundation [grant No. PHY-1307153]; and the Welch Foundation [grant No. A1547]. We thank Anton Shutov for assistance. M.S. is supported by the Herman F. Heep and Minnie Belle Heep Texas A \& M University Endowed Fund administered by the Texas A \& M Foundation. A. Z. gratefully acknowledges her Diversity Fellowship from Texas A \& M University.

\section{References}

[1] L. Allen, M. J. Padgett, M. Babiker, The Orbital Angular Momentum Of Light, Progress in Optics 39 (1999) 291-372. doi:10.1016/ S0079-6638(08)70391-3.

[2] M. S. Kirilenko, S. N. Khonina, Information transmission using optical 1. vortices, Optical Memory and Neural Networks 22 (2) (2013) 81-89. doi: 10.3103/S1060992X13020069

URL http://link. springer.com/10.3103/S1060992X13020069

[3] S. N. Khonina, N. L. Kazanskiy, V. A. Soifer, Optical Vortices in a Fiber : Mode Division Multiplexing and Multimode Self-Imaging, Recent Progress in Optical Fiber Research (2012) 327-352.

[4] S. Berdagué, P. Facq, Mode division multiplexing in optical fibers., Applied Optics 21 (11) (1982) 1950-1955. doi:10.1364/A0.21.001950. 
[5] K. T. Gahagan, G. A. Swartzlander, Optical vortex trapping of particles, Optics Letters 21 (11) (1996) 827-829. arXiv:arXiv:1011.1669v3, doi: 10.1364/OL.21.000827.

[6] D. G. Grier, A revolution in optical manipulation, Nature 424 (2003) 810816. doi:10.1038/nature01935.

[7] M. Dienerowitz, M. Mazilu, K. Dholakia, Optical manipulation of nanoparticles: a review, Journal of Nanophotonics 2 (September) (2008) 1-32. doi:10.1117/1.2992045.

[8] G. Foo, D. M. Palacios, G. A. Swartzlander Jr, Optical vortex coronagraph, Optics Letters 30 (24) (2005) 3308-3310. doi:10.1364/0L.30.003308 URL http://www.opticsinfobase.org/abstract.cfm?URI= OL-30-24-3308\$ddelimiter"026E30F\$npapers3://publication/ doi/10.1364/OL.30.003308

[9] D. P. Ghai, S. Vyas, P. Senthilkumaran, R. S. Sirohi, Detection of phase singularity using a lateral shear interferometer, Optics and Lasers in Engineering 46 (6) (2008) 419-423. doi:10.1016/j.optlaseng.2008.02.001.

[10] H. I. Sztul, R. R. Alfano, Double-slit interference with Laguerre-Gaussian 口 beams, Optics Letters 31 (7) (2006) 999-1001. doi:10.1364/0L.31. 000999 .

[11] A. G. White, C. P. Smith, N. R. Heckenberg, H. Rubinsztein-Dunlop, R. McDuff, C. O. Weiss, C. Tamm, Interferometric measurements of phase singularities in the output of a visible laser, Journal of Modern Optics 38 (12) (1991) 2531-2541.

[12] C.-S. Guo, L.-L. Lu, H.-T. Wang, Characterizing topological charge of optical vortices by using an annular aperture, Optics Letters 34 (23) (2009) 3686-3688. doi:10.1364/OL.34.003686.

URL http://www.opticsinfobase.org/abstract.cfm?id=190633 
[13] L. Yongxin, T. Hua, P. Jixiong, L. Baida, Detecting the topological charge of vortex beams using an annular triangle aperture, Optics and Laser Technology 43 (7) (2011) 1233-1236. doi:10.1016/j.optlastec.2011.03. 015.

[14] S. N. Khonina, V. V. Kotlyar, V. a. Soifer, K. Jefimovs, P. Pääkkönen, J. Turunen, Astigmatic Bessel laser beams, Journal of Modern Optics 51 (5) (2004) 677-686. doi:10.1080/09500340408235545.

- URL http://www.tandfonline.com/doi/abs/10.1080/ 09500340408235545

[15] V. V. Kotlyar, S. N. Khonina, A. a. Almazov, V. a. Soifer, K. Jefimovs, J. Turunen, Elliptic Laguerre-Gaussian beams., Journal of the Optical Society of America A, Optics, image science, and vision 23 (1) (2006) 43-56. doi:10.1364/JOSAA.23.000043. URL http://www.ncbi.nlm.nih.gov/pubmed/16478059

[16] A. A. Almazov, S. N. Khonina, V. V. Kotlyar, How the tilt of a phase diffraction optical element affects the properties of shaped laser beams matched with a basis of angular harmonics, Journal of Optical Technology 73 (9) (2006) 633-639.

[17] E. Abramochkin, V. Volostnikov, Beam transformations and nontransformed beams, Optics Communications 83 (1-2) (1991) 123-135. doi: 10.1016/0030-4018(91)90534-K.

[18] M. W. Beijersbergen, L. Allen, H. E. L. O. van der Veen, J. P. Woerdman, Astigmatic laser mode converters and transfer of orbital angular 265 momentum, Optics Communications 96 (1993) 123-132. doi:10.1016/ 0030-4018(93) 90535-D.

[19] V. Denisenko, V. Shvedov, A. S. Desyatnikov, D. N. Neshev, W. Krolikowski, A. Volyar, M. Soskin, Y. S. Kivshar, Determination of topological 
charges of polychromatic optical vortices., Optics Express 17 (26) (2009) 23374-23379. doi:10.1364/OE.17.023374.

[20] P. Vaity, J. Banerji, R. Singh, Measuring the topological charge of an optical vortex by using a tilted convex lens, Physics Letters A 377 (15) (2013) 1154-1156. doi:10.1016/j.physleta.2013.02.030. S0375960113001953

[21] T. R. Harvey, B. J. McMorran, A Stern-Gerlach-like approach to eleca tron orbital angular momentum measurement, arXiv:1606.0361arXiv: 1606.03631 .

URL http://arxiv.org/abs/1606.03631

[22] H. Ma, H. Hu, W. Xie, X. Xu, Study on the generation of a vortex laser beam by using phase-only liquid crystal spatial light modulator, Optical Engineering 52 (9) (2013) 091721. doi:10.1117/1.0E.52.9.091721. URL http://opticalengineering.spiedigitallibrary.org/article. aspx?doi=10.1117/1.0E.52.9.091721

[23] K. Bezuhanov, A. Dreischuh, G. G. Paulus, M. G. Schätzel, H. Walther, Vortices in femtosecond laser fields, Optics Letters 29 (16) (2004) 19421944. doi:10.1364/OL.29.001942,

[24] S. B. Cavalcanti, G. P. Agrawal, M. Yu, Noise amplification in dispersive nonlinear media, Physical Review A 51 (5) (1995) 4086-4092.

[25] M. V. Vasnetsov, K. Staliunas, Optical Vortices (Horizons in World Physics Volume 228), Nova Science Publishers, Inc., 1999.

[26] V. Jarutis, A. Matijošius, V. Smilgevičius, A. Stabinis, Second harmonic generation of higher-order Bessel beams, Optics Communications 185 (1-3) (2000) 159-169. doi:10.1016/S0030-4018(00)00974-3.

[27] A. V. Gorbach, D. V. Skryabin, Cascaded generation of multiply charged optical vortices and spatiotemporal helical beams in a Raman medium, 
[28] M. Zhi, K. Wang, X. Hua, H. Schuessler, J. Strohaber, A. V. Sokolov,

[29] J. Strohaber, J. Abul, M. Richardson, F. Zhu, A. A. Kolomenskii, H. A.

[30] P. Hansinger, G. Maleshkov, I. L. Garanovich, D. V. Skryabin, D. N. Ne-

[32] A. Bekshaev, M. Soskin, M. Vasnetsov, Paraxial Light Beams with Angular Momentum, arXiv:0801.2309 arXiv:0801.2309.

URL http://arxiv.org/abs/0801.2309

[33] D. Rozas, C. Law, G. Swartzlander, Propagation dynamics of optical vordoi:10.1364/JOSAB.14.003054.

URL http: //www . opticsinfobase . org/abstract . cfm?id=35222

[34] S. Khonina, V. Kotlyar, Generation and selection of laser beams represented by a superposition of two angular harmonics, Journal of Modern 
Optics 51 (5) (2004) 761-773. doi:10.1080/09500340408235551.

$\square$

URL

http://www.tandfonline.com/doi/abs/10.1080/

09500340408235551

[35] S. N. Khonina, V. V. Kotlyar, V. A. Soifer, P. Pääkkönen, J. Simonen,

J. Turunen, An analysis of the angular momentum of a light field in terms of angular harmonics, Journal of Modern Optics 48 (10) (2001) 1543-1557. doi:10.1080/09500340110047501.

URL http://www .informaworld.com/index/714043941.pdf

[36] S. N. Khonina, V. V. Kotlyar, V. A. Soifer, P. Paakkonen, J. Turunen, Measuring the light field orbital angular momentum using DOE, Optical Memory and Neural Networks 9 (4) (2001) 241-255.

[37] V. V. Kotlyar, S. N. Khonina, V. A. Soifer, Light field decomposition in angular harmonics by means of diffractive optics, Journal of Modern Optics 45 (7) (1998) 1495-1506. doi:10.1080/09500349808230644.

URL http://www.tandfonline.com/doi/abs/10.1080/ 09500349808230644

[38] J. Leach, M. Padgett, S. Barnett, S. Franke-Arnold, J. Courtial, Measuring the Orbital Angular Momentum of a Single Photon, Physical Review Letters 88 (25) (2002) 257901. doi:10.1103/PhysRevLett.88.257901. URL http://link.aps.org/doi/10.1103/PhysRevLett.88.257901

[39] A. Y. Bekshaev, M. S. Soskin, M. V. Vasnetsov, Transformation of higherorder optical vortices upon focusing by an astigmatic lens, Optics Communications 241 (4-6) (2004) 237-247. doi:10.1016/j.optcom.2004.07.023

[40] R. S. Sirohi, A course of experiments with He-Ne Laser, New Age International, 1991. 